\title{
Myelofibrosis: the present and the future-a review article
}

\section{Introduction}

Myelofibrosis (MF) is a myeloproliferative neoplasm characterized by symptoms mainly derived from anemia and splenomegaly and associated with a median survival of around 6 years. Allogeneic stem cell transplantation (allo-SCT) remains the only curative therapy of MF but is applicable to a minority of patients. Discovery of the JAK2 mutation has provided the basis for the introduction of a new class of drugs, the JAK inhibitors, in the treatment of MF.

\section{Therapeutic approaches}

Currently, there is no drug option that is curative. The goal for most patients is to relieve symptoms, reduce an enlarged spleen, improve blood cell counts and minimize the risk of complications. Myelofibrosis is a disease with few approved treatment options, but many new agents are being investigated and developed. For highrisk disease, stem cell transplant is often considered. The standard of care for myelofibrosis is ruxolitinib (Jakafi, Incyte), which is the only therapy approved by the US Food and Drug Administration for the disease. Ruxolitinib inhibits JAK1 and JAK2, and it can be effective regardless of which molecular mutations a patient has. The data show that ruxolitinib will improve splenomegaly in most patients. In approximately half of patients, this improvement will meet the response criteria from the International Working Group for Myelofibrosis. Most patients will also experience improvement in other symptoms. Additionally, clinical trials demonstrate that ruxolitinib increases survival, when accounting for crossover. That being said, ruxolitinib is not considered a curative therapy. A limitation of ruxolitinib is that it typically does not improve anemia or thrombocytopenia. Ruxolitinib has been tried in at least 10 different combinations to date. Those studies are largely ongoing, so there are no final conclusions. At this time, there are no clear data to support the use of a particular combination outside of a clinical trial.

Pacritinib is an oral tyrosine kinase inhibitor (TKI) with activity against JAK2 and FMS-like tyrosine kinase 3 (FLT3). Results from the phase 3 PERSIST-1 trial (A Randomized Controlled Phase 3 Study of Oral Pacritinib Versus Best Available Therapy in Patients with Primary Myelofibrosis, Post-Polycythemia Vera Myelofibrosis, or Post-Essential Thrombocythemia Myelofibrosis) were positive when compared with best alternative therapy among patients with myelofibrosis who had not received previous treatment with JAK2 inhibitors. The trial enrollment criteria had no minimum threshold for the platelet count, so the population included patients with high blast counts and with very advanced disease moving toward acute leukemia. A phase 2 study, PERSIST-2, was evaluating pacritinib as second-line therapy. In February 2016, the FDA placed a full clinical hold on pacritinib in order to more closely examine the deaths that occurred among patients in the trial. It is not clear that the deaths seen on the study are a side effect of pacritinib, and the toxicity profile had been favorable. Ongoing analysis is attempting to discern whether the deaths could be attributable to adverse patient selection, or whether they represent a true safety concern with pacritinib.

Two important phase 3 studies are comparing ruxolitinib vs momelotinib, with the aim of improving anemia. SIMPLIFY-1
Volume 10 Issue 2 - 2019

\author{
Hajj Adel Anis \\ Cedars Jebel Ali International Hospital, UAE
}

Correspondence: Hajj Adel Anis, Medical Oncologist at Cedars Jebel Ali International Hospital, 9370 Rue Lajeunesse, Montreal, UAE, Tel 438992 5516, Email ahajj@dr.com

Received: January 30, 2019 | Published: March 20, 2019

(A Phase 3, Randomized, Double-Blind Active-Controlled Study Evaluating Momelotinib vs Ruxolitinib in Subjects With Primary Myelofibrosis (PMF) or Post-Polycythemia Vera or Post-Essential Thrombocythemia Myelofibrosis (Post-PV/ET MF) is for first line therapy and SIMPLIFY-2 (A Phase 3, Randomized Study to Evaluate the Efficacy of Momelotinib vs Best Available Therapy in Anemic or Thrombocytopenic Subjects With Primary Myelofibrosis, PostPolycythemia Vera Myelofibrosis, or Post-Essential Thrombocythemia Myelofibrosis Who Were Treated With Ruxolitinib) is for secondline therapy. There are several studies in patients with relapsed or refractory disease after treatment with ruxolitinib. A phase 2 trial is evaluating imetelstat, a telomerase inhibitor, at doses found to have activity in a phase 1 trial. Another phase 2 study is evaluating PRM151 , an antifibrosing agent. ${ }^{1-7}$

\section{Future perspectives}

Investigators are evaluating whether early intervention can reduce the risk of progression. There are also efforts to understand how the interactions of molecular mutations can impact patient progression, and whether these mechanisms can be targeted in a more effective way. Much research is focused on patients with advanced disease who are moving toward acute leukemia.

\section{References}

1. Pardanani A, Tefferi A. Targeting myeloproliferative neoplasms with JAK inhibitors. Curr Opin Hematol. 2011;18(2):105-110.

2. Verstovsek S. Janus-activated kinase 2 inhibitors: a new era of targeted therapies providing significant clinical benefit for Philadelphia chromosome-negative myeloproliferative neoplasms. J Clin Oncol. 2011;29(7):781-783.

3. Quintás-Cardama A, Verstovsek S. Spleen deflation and beyond: the pros and cons of Janus kinase 2 inhibitor therapy for patients with myeloproliferative neoplasms. Cancer. 2012 Feb;118(4):870-877.

4. Cervantes F, Mesa R, Harrison C. JAK inhibitors: beyond spleen and symptoms? Haematologica. 2013;98(2):160-162.

5. Pardanani A, Laborde RR, Lasho TL, et al. Safety and efficacy of CYT387, a JAK1 and JAK2 inhibitor, in myelofibrosis. Leukemia. 2013;27(6):1322-1327.

6. Vainchenker W, Favale F. Myelofibrosis, JAK2 inhibitors and erythropoiesis. Leukemia. 2013;27(6):1219-1223.

7. Verstovsek S. Therapeutic potential of Janus-activated kinase-2 inhibitors for the management of myelofibrosis. Clin Cancer Res. 2010;16(7):1988-1996. 